\title{
Nivel de conocimiento y práctica de medidas de bioseguridad: Hospital San José, 2016
}

Frank Dennys Tamariz Chavarria ${ }^{1, a}$

RESUMEN

Objetivo: Determinar la relación entre el nivel de conocimiento y la práctica de bioseguridad del personal de salud en los servicios de hospitalización de Medicina, Cirugía, Ginecología y Pediatría del Hospital San José del Callao. Materiales y métodos: Estudio cuantitativo, observacional, descriptivo, transversal en una población de 100 trabajadores de salud de los servicios de hospitalización de Medicina, Cirugía, Ginecología y Pediatría del Hospital San José, de enero a junio del 2016.

Resultados: El nivel de conocimiento del personal de salud es de medio (55\%) a bajo (19 \%), que es una cifra alarmante, ya que la población a estudiar labora en las áreas de hospitalización. El nivel de práctica del personal de salud es bueno (65\%), pero con riesgo a desviarse a un nivel inferior desfavorable en las áreas de hospitalización. Conclusiones: El nivel de conocimiento y la práctica de bioseguridad del personal de salud presentaron una relación significativa entre ambas variables (prueba exacta de Fisher $\mathrm{p}=.000$ ), lo cual significa que es necesario el conocimiento sobre bioseguridad para una práctica favorable, y así disminuir el riesgo de infecciones intrahospitalarias.

Palabras clave: Conocimiento; Salud ocupacional; Medidas de seguridad; Personal de salud (Fuente: DeCS BIREME).

\section{Level of knowledge and practice of biosafety measures at the Hospital San José, 2016}

\section{ABSTRACT}

Objective: To determine the relationship between the level of knowledge and the practice of biosafety measures among the health personnel from the inpatient services of internal medicine, surgery, gynecology and pediatrics of the Hospital San José del Callao.

Materials and methods: A quantitative, observational, descriptive, cross-sectional study conducted in a population of 100 health workers from the inpatient services of internal medicine, surgery, gynecology and pediatrics of the Hospital San José from January to June 2016.

Results: The level of knowledge among the health personnel ranges from medium (55\%) to low (19\%). These are alarming figures, since the study population works in inpatient departments. The level of practice among the health personnel is good (65\%), but it is at risk of changing to an unfavorable lower level in the inpatient departments.

Conclusions: The level of knowledge and practice of biosafety measures among the health personnel showed a significant relationship between both variables (Fisher's exact test $p=.000$ ), which means that knowledge about biosafety measures is necessary for a favorable practice, and thus decrease the risk of nosocomial infections.

Keywords: Knowledge; Occupational health; Security measures; Health personnel (Source: MeSH NLM).

1. Hospital Guillermo Almenara Irigoyen. Lima, Perú.

a. Médico Especialista Administración en Salud. 


\section{INTRODUCCIÓN}

La bioseguridad debe entenderse como una doctrina de comportamiento encaminada a lograr actitudes y conductas que disminuyan el riesgo del trabajador de la salud de adquirir infecciones en el medio laboral. Compromete también a todas aquellas otras personas que se encuentran en el ambiente asistencial, el cual debe estar diseñado en el marco de una estrategia de disminución de riesgos ${ }^{(1)}$.

La presente investigación se enfoca en las medidas de bioseguridad observadas por el personal de salud del Hospital San José del Callao, y su importancia en relación al nivel de conocimiento y práctica de las mismas para el autocuidado del personal y la prevención de enfermedades e infecciones intrahospitalarias, que pueden prevenirse mediante la práctica de las medidas de bioseguridad. Además, son de suma importancia cuando pensamos en la gestión de la calidad.

Según Espinosa-Aquino B (2010), la definición de bioseguridad se deduce a partir de sus componentes: "bio" (del griego bios), que significa vida, y "seguridad", que proviene de estar seguro, libre de daño o peligro. Por lo tanto, bioseguridad puede entenderse como la calidad de la vida que está libre de daño, riesgo o peligro (2).

El concepto de bioseguridad origina distintas afirmaciones, una de ellas, la de Franco A (2005), plantea que para conseguir actos clínicos más seguros y sin errores, es indispensable una cultura institucional de seguridad del paciente, prácticas seguras y trabajo constante para mejorar al grupo humano ${ }^{(3)}$.

Según el Manual de Bioseguridad de la Universidad Industrial de Santander de Colombia (2012), se define la bioseguridad como el conjunto de medidas preventivas, destinadas a mantener el control de factores de riesgo laborales procedentes de agentes biológicos, físicos o químicos, logrando la prevención de impactos nocivos, asegurando que el desarrollo o producto final de dichos procedimientos no atenten contra la salud y seguridad de trabajadores de la salud, pacientes y el medio ambiente (4).

Así mismo, Bautista R (2013), afirma que las normas de bioseguridad tienen un rol preventivo para proteger la salud y reducir la posibilidad de transmisión de microorganismos presentes en los ambientes hospitalarios en donde deben ser implantadas ${ }^{(5)}$.

Para prevenir la adquisición de enfermedades infectocontagiosas relacionadas con el trabajo del personal es fundamental implementar medidas de buenas prácticas de bioseguridad. El trabajador tiene el derecho a conocer los riesgos existentes en su lugar de trabajo y es, en última instancia, el responsable de cumplir las medidas de bioseguridad instauradas en la institución ${ }^{(6)}$.

Asimismo, Fink S (2010) refiere que deben respetarse las normas de bioseguridad en todos los ámbitos de atención de salud humana, animal y de investigación, y realizar prácticas seguras para disminuir el potencial de exposición a riesgo de tipo biológico ${ }^{(7)}$.

Castells S (2003) señala que el hecho de existir desconocimiento sobre las precauciones para manipular las muestras de sangre, la ropa contaminada y objetos punzocortantes, aunque sea en una pequeña parte del personal de enfermería, demuestra una debilidad del aprendizaje en los enfermeros de nueva incorporación y deficiencia en la educación permanente y continuada de los servicios donde laboran, ya que el conocimiento de estas precauciones son un requisito casi obligatorio para este personal, por estar presentes en su proceder diario ${ }^{(8)}$. Del Valle S (2002) afirma que el uso de normas efectivas de control y prevención, así como las medidas de protección universal permitirán evitar la contaminación cruzada entre pacientes, el personal auxiliar del consultorio y hasta de pacientes al profesional de salud ${ }^{(9)}$.

Los resultados de la investigación en su doble vertiente, desde la cuantificación de las percepciones y la profundización en el mundo de los significados, permitirá contar con información teórica que servirá como punto de referencia para diseñar algunas propuestas revitalizadoras en el comportamiento laboral que conduzcan, en el mediano plazo, a la construcción progresiva de una cultura de bioseguridad para el personal de salud.

\section{MATERIALES Y MÉTODOS}

El estudio realizado fue de enfoque cuantitativo, observacional, descriptivo, transversal y prospectivo. La población objetivo estuvo constituida por todo el personal asistencial de los servicios de hospitalización de Medicina, Cirugía, Ginecología y Pediatría del Hospital San José.

La muestra de estudio estuvo conformada por el personal de salud, 31 médicos, 33 enfermeras y 36 técnicos de enfermería de ambos sexos, en un total de 100, comprendidos durante el periodo de enero a junio del 2016.

Se utilizó un cuestionario, que se distribuyó al personal de salud en su respectivo turno de trabajo, para medir el nivel de conocimiento de medidas de bioseguridad de riesgos biológicos.

Para la observación se utilizó una lista de cotejo, cuyos 
datos recolectaron a través de la observación directa a cada miembro del personal de salud de los servicios incluidos en la investigación, a fin de verificar, si se aplican o no las medidas preventivas de los riesgos biológicos. El seguimiento se realizó durante siete días consecutivos en el turno de mañana, por ser en el que es mayor la congestión de trabajo.

La información se organizó en una matriz de Excel para su posterior análisis e interpretación en el paquete estadístico para ciencias sociales, SPSS, versión 20; cuantitativamente se usó estadística descriptiva: media para las variables cuantitativas y mediana para los datos cualitativos. El nivel de significancia de los resultados se estableció mediante la prueba del Chi-cuadrado. Los resultados se ilustraron mediante tabulación simple cruzada y gráfica respectiva, de acuerdo a los objetivos propuestos en el trabajo.

\section{RESULTADOS}

En el presente estudio la muestra estuvo conformada por 100 trabajadores de salud de los servicios de hospitalización de Medicina, Cirugía, Ginecología y Pediatría del Hospital San José, obteniendo los siguientes resultados:

El nivel de conocimiento sobre bioseguridad del personal de salud es, en la mayoría de los casos de medio (55\%) a bajo (19\%), lo que es alarmante, ya que la población comprendida en el estudio labora en las áreas de hospitalización (Figura 1).

Figura 1. Nivel de conocimiento sobre bioseguridad del personal asistencial en los servicios de hospitalización Hospital San José - Callao 2016

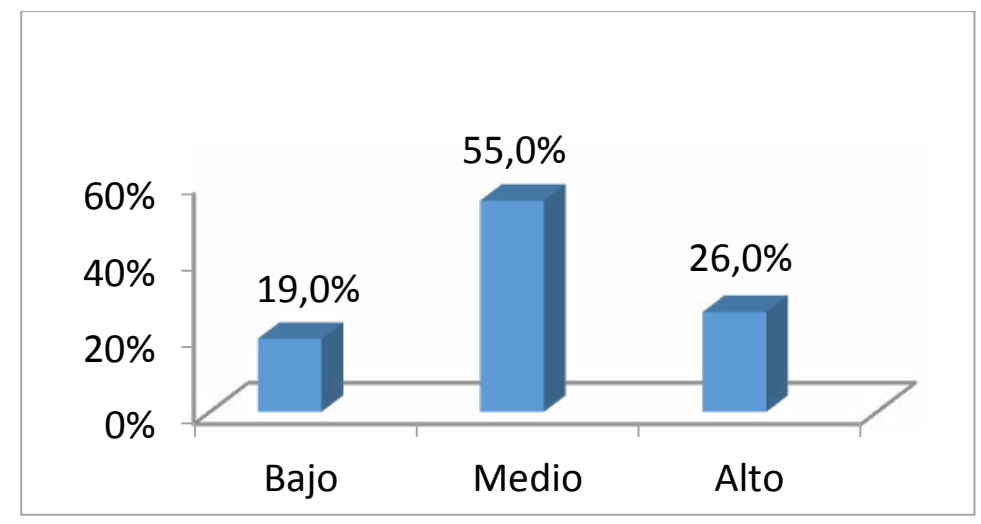

En la figura 2 se presenta el nivel de práctica de las medidas de bioseguridad por el personal de salud. El nivel es bueno (65\%), pero se observa un riesgo a desviarse a un nivel inferior desfavorable en las áreas de hospitalización.

Figura 2. Nivel de práctica sobre las medidas de bioseguridad del personal asistencial en los servicios de hospitalización Hospital San José - Callao 2016

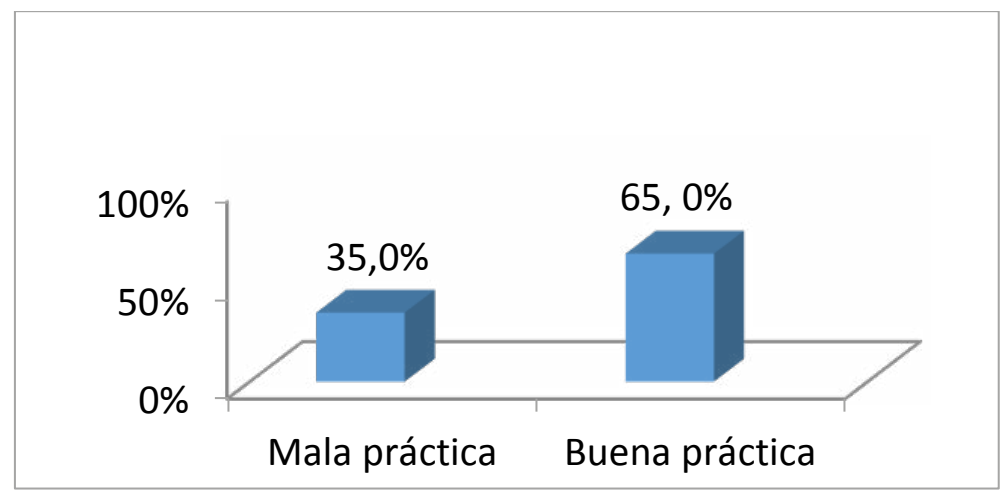


De acuerdo a la figura 3 la relación entre nivel de conocimientos y práctica de bioseguridad del personal hospitalario es altamente significativa (prueba exacta de Fisher: ${ }^{* * *} p=.000$ ). También se observa que cuando el grado de conocimientos sobre bioseguridad es medio/alto, la práctica de los mismos se realiza en $64 \%$ de los casos (nivel favorable); por otro lado, cuando los conocimientos están en un grado bajo, las prácticas de bioseguridad descienden a $18 \%$ (nivel deficiente).

Figura 3. Relación entre el nivel de conocimiento y de práctica sobre bioseguridad del personal asistencial en los servicios de hospitalización Hospital San José - Callao 2016

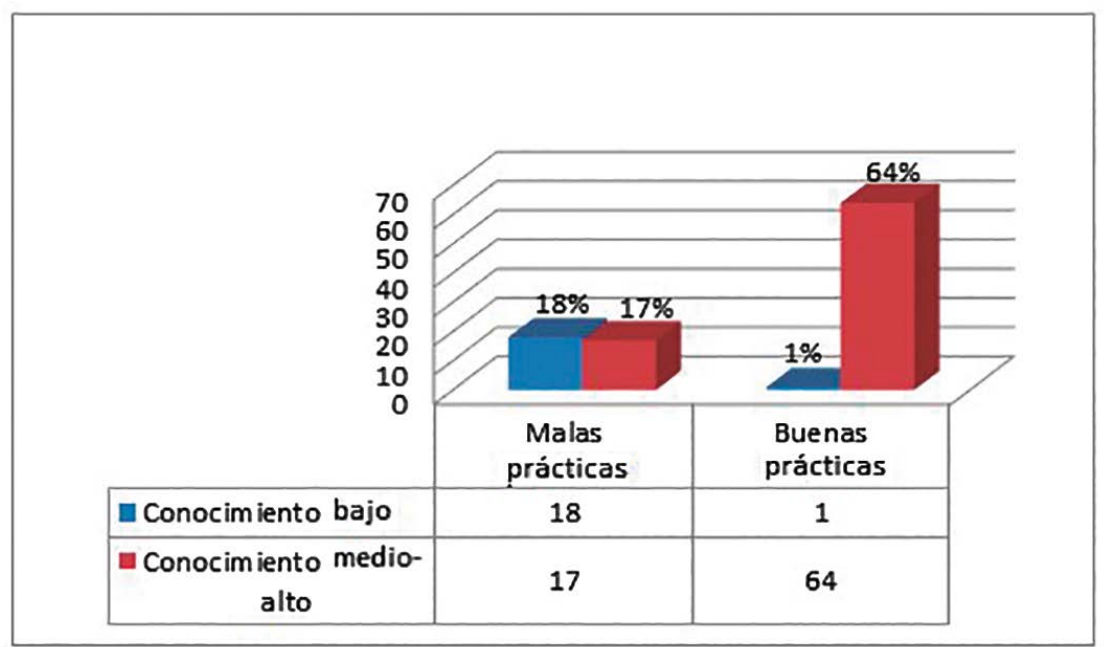

Los conocimientos sobre riesgo biológico y su relación con el lavado de manos se muestran en la figura 4. Ambas variables tienen una asociación altamente significativa (según la prueba exacta de Fisher: ${ }^{* * *} p=.000$ ). También observamos que cuando los conocimientos sobre riesgo biológico alcanzan un nivel medio/alto, las prácticas de lavado de manos son de buen nivel (57\%). Por otro lado, si este conocimiento es menor, entonces aumentan las malas prácticas en el lavado de manos.

Figura 4. Relación entre el nivel de conocimiento sobre riesgo biológico y el nivel de práctica sobre lavado de manos del personal asistencial del Hospital San José - Callao 2016

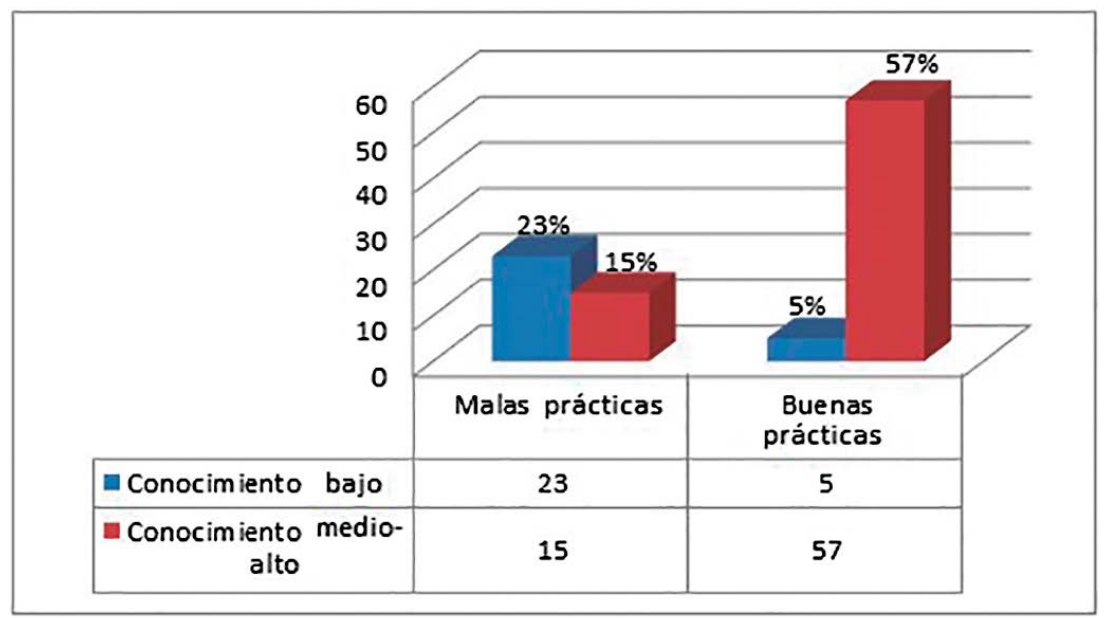


Igualmente, observamos que cuando los conocimientos sobre medidas de bioseguridad alcanzan un nivel alto, las prácticas sobre uso de barreras protectoras son adecuadas (20\%); por el contrario, si los conocimientos sobre medidas de bioseguridad son de nivel bajo - medio, aumenta la ineficiencia en el empleo de barreras protectoras (40\%) (Figura 5). Además, ambas variables tienen una asociación altamente significativa (prueba exacta de Fisher: ${ }^{* * *} \mathrm{p}=.000$ ).

Figura 5. Relación entre el nivel de conocimiento sobre medidas de bioseguridad y el nivel de práctica sobre el uso de barreras protectoras del personal asistencial del Hospital San José - Callao

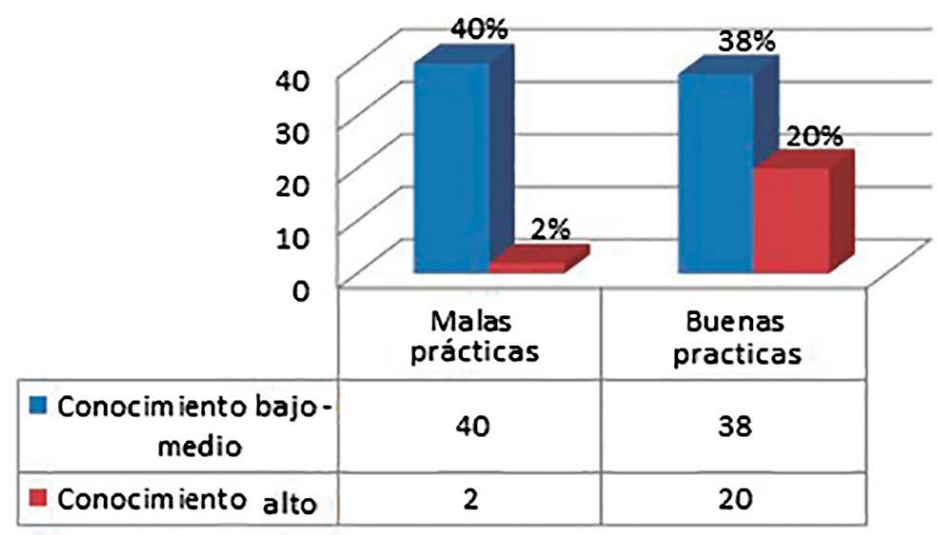

En la figura 6 se muestra que el conocimiento sobre el manejo de residuos hospitalarios no está asociado con las prácticas de manejo de residuos sólidos, siendo no significativo (Chi-cuadrado: $p=.210$ ). Además, observamos que cuando los conocimientos sobre manejo de residuos hospitalarios son de nivel medio, las prácticas de manejo de residuos sólidos son de nivel adecuado (49\%); pero si están en un nivel bajo, el manejo de residuos sólidos es deficiente.

Figura 6. Relación entre el nivel de conocimiento sobre manejo de residuos hospitalarios y el nivel de práctica sobre manejo de residuos sólidos del personal asistencial

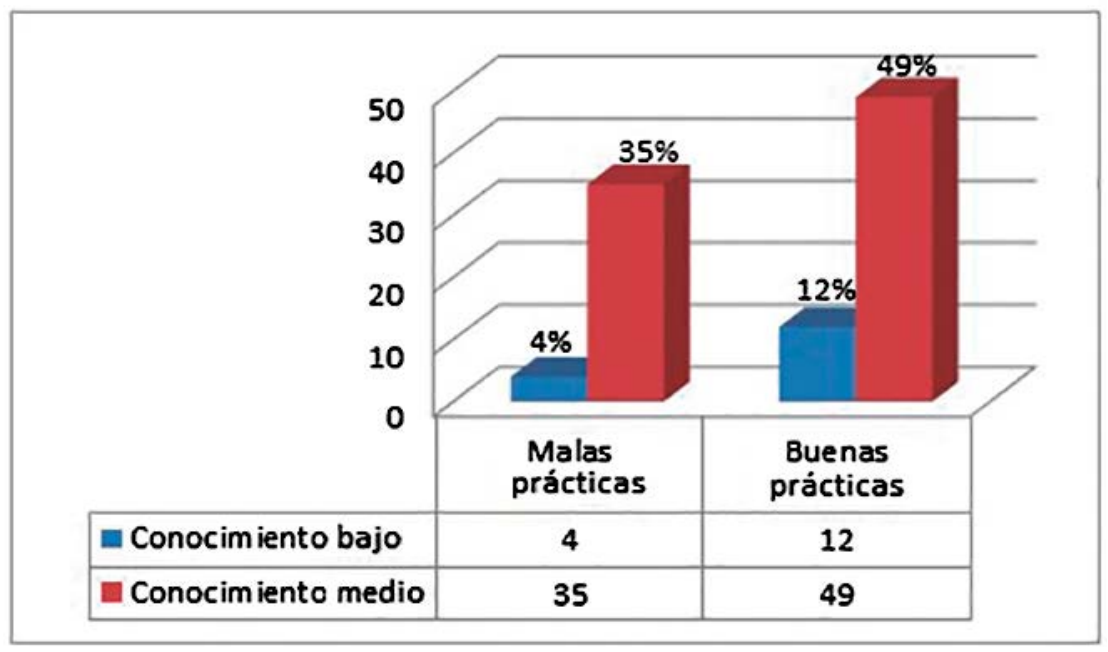




\section{DISCUSIÓN}

La base de la práctica de la bioseguridad es la evaluación del riesgo que implica llevar a cabo un procedimiento o un experimento. A pesar de contar con otras herramientas para el mismo fin, lo más importante sigue siendo el juicio profesional ${ }^{(10)}$.

Podemos afirmar que el conocimiento sobre bioseguridad en el personal asistencial de los servicios de hospitalización fue de nivel medio con $55 \%$, nivel alto con $26 \%$ y nivel bajo con $19 \%$. Los resultados que se obtuvieron se asemejan a los encontrados por Huamán D (2013) en su investigación "Nivel de conocimiento y práctica de medidas de bioseguridad en las enfermeras de los servicios de medicina del Hospital Belén de Trujillo 2014", quien encontró que el $56 \%$ de enfermeras tuvo un nivel de conocimiento medio, el $44 \%$ obtuvo un nivel de conocimiento alto y ninguna registró un nivel bajo ${ }^{(11)}$.

El nivel de conocimientos del personal de salud (medio a bajo) representa una situación alarmante porque es el grupo que se encuentra más propenso a adquirir infecciones intrahospitalarias, así como transmitirlas, lo que conlleva a un posible aumento en la incidencia de este cuadro, lo que se refleja en indicadores hospitalarios como días de hospitalización/paciente. Además, aumenta la estancia hospitalaria del enfermo y sobrecarga la economía intrahospitalaria.

Por lo tanto, el personal de salud exige una mayor capacitación, reforzando los conocimientos de bioseguridad para todo procedimiento que se realice. Por tal motivo, las medidas de bioseguridad que se tomen serán más estrictas cuanto más peligrosos sean los gérmenes que se manejan en el área de trabajo ${ }^{(12)}$.

Podemos afirmar que las prácticas de las medidas de bioseguridad en el personal asistencial de los servicios de hospitalización del centro San José del Callao, en 2016 fueron buenas en el $65 \%$ de los casos y malas, en el $35 \%$, lo que coincide con la investigación ya mencionada de Huamán D (2013), en la que $72 \%$ de enfermeras realizan buenas prácticas de bioseguridad y $28 \%$, lo contrario.

El nivel de práctica de medidas de bioseguridad es adecuado, pero es necesario disminuir la incidencia de malos procedimientos Por tal motivo, es importante la difusión, entre el personal sanitario, de medidas de bioseguridad y normas de comportamiento y de manejo preventivo de microorganismos potencialmente patógenos, con el propósito de disminuir la probabilidad de adquirir infecciones en el medio laboral (13).

Los conocimientos y la práctica de las medidas de bioseguridad están estrechamente asociados, ambas variables muestran una asociación altamente significativa (prueba exacta de Fisher: ${ }^{* * *} \mathrm{p}=.000$ ). También observamos que cuando los conocimientos sobre bioseguridad están en una escala medio/alta, las prácticas sobre bioseguridad alcanzan un buen nivel (64\%); así mismo, si las nociones de bioseguridad son deficientes, las prácticas llegan a niveles bajos (18\%).

Los resultados de nuestra investigación son similares a los de Huamán D (2013) en la investigación ya mencionada quien encontró una relación entre quienes tienen un conocimiento alto (44\%) en bioseguridad y realizan buenas prácticas, los que tiene un conocimiento medio (56 $\%$ ) realizaron buenas prácticas en $28 \%$ y malas prácticas igualmente en $28 \%$ de los casos, con una relación significativa de 0.006 .

En conclusión, a mayor nivel de conocimiento por parte del personal de salud, mejor será la práctica de medidas de bioseguridad durante los procedimientos hospitalarios. Por lo tanto, es indispensable la variable conocimiento en la prevención de infecciones nosocomiales. Además, es preciso tomar acciones como la capacitación constante y monitoreo de actividades, el empleo de manuales y protocolos de procedimientos, el uso de equipos de protección y mejorar las estrategias sanitarias institucionales. Debido a que hay un compromiso de las personas que se encuentran en el ambiente asistencial, este debe estar diseñado en el marco de una estrategia de disminución de riesgos, incorporando los tres principios fundamentales ${ }^{(14)}$.

Por consiguiente, este conjunto de medidas deben aplicarse sistemáticamente a todos los pacientes sin distinción, considerando que toda persona puede ser de alto riesgo; asimismo, identificar todo fluido corporal como potencialmente contaminante ${ }^{(15)}$.

El concepto de riesgo biológico está ligado a las prácticas de lavado de manos; la asociación de ambas variables es altamente significativa (prueba exacta de Fisher: ${ }^{* * *} \mathrm{p}=.000$ ). De esta manera, si los conocimientos acerca de riesgo biológico son de nivel medio/alto, las prácticas sobre lavado de manos son óptimas (57\%); por otro lado, si el nivel de conocimientos es bajo, también lo son las prácticas de lavado de manos (23\%). Por lo tanto, un mayor conocimiento de riego biológico implica una mejor práctica del lavado de manos y, en consecuencia, la reducción de accidentes biológicos. Además, toda la población debe cumplir el principio de universalidad, ya sea que presente o no patologías ${ }^{(16)}$.

Del mismo modo, el conocimiento de las medidas de bioseguridad se relaciona con el uso de barreras protectoras (asociación altamente significativa según prueba exacta de Fisher: ${ }^{* * *} \mathrm{p}=0.000$ ). Por lo mismo, si se tiene una noción 
adecuada de las medidas de bioseguridad (nivel alto) se consigue un buen nivel en el uso de barreras protectoras (20\%), pero si los conocimientos sobre medidas de bioseguridad son de nivel bajo / medio, las prácticas sobre uso de barreras protectoras son de nivel inadecuado (40\%). Así, podemos señalar que en un conocimiento adecuado sobre medidas de bioseguridad conlleva a una disminución notable de casos de mala práctica en el uso de barreras protectoras, mientras que un nivel de conocimiento medio/bajo, existe riesgo de incidencia de casos de mala práctica.

Los conocimientos sobre manejo de residuos hospitalarios no están asociados con las prácticas de manejo de residuos sólidos, (no significativo según Chi-cuadrado: $p=.210$ ). También observamos que, tal como ocurre en otros casos, a mejor nivel de conocimiento de bioseguridad, el manejo de residuos sólidos es óptimo, y que, si los conocimientos sobre manejo de residuos hospitalarios son de nivel medio, las prácticas de manejo de residuos sólidos son de buen nivel (49\%). Esto es importante si tenemos en cuenta que una adecuada manipulación de los mismos es indispensable para reducir el riesgo de exposición de sangre y líquidos ${ }^{(15)}$. Así también, observamos que cuando los conocimientos sobre manejo de residuos hospitalarios son de nivel medio, el manejo de residuos sólidos es deficiente (35\%). Se concluye que no existe relación entre los conocimientos sobre manejo de residuos hospitalarios y las prácticas de manejo de residuos sólidos en el personal asistencial de los servicios de hospitalización del Hospital San José del Callao en 2016.

Una cultura de bioseguridad difundida y priorizada en el personal de salud en relación con el lavado de manos, constituye una barrera protectora trascendental ya que evita que el personal de salud contraiga gérmenes a través de las manos ${ }^{(17)}$. Además, es el método más eficiente para disminuir el traspaso de material infectante de un individuo a otro y cuyo propósito es la reducción continua de la flora residente y desaparición de la flora transitoria de la piel ${ }^{(18)}$.

Es relevante destacar la educación y capacitación continua del personal médico y no médico como única manera, a través de la comprensión, de estimular el cumplimiento de las normas de bioseguridad. Debe remarcarse que estas medidas tienden no solo a la prevención de la diseminación entre los pacientes, sino también a la protección del personal y su familia ${ }^{(18)}$.

La bioseguridad como parte constitutiva de la prevención ha de integrarse en el sistema de gestión de toda institución, incluyéndola en todos los niveles de organización, en todas las actividades con riesgo de accidente biológico que se realicen y ordenen y en todas las decisiones que se adopten. Esta integración ha de ser patente en todo el proceso de gestión y estar contemplada en el plan de prevención de riesgos laborales ${ }^{(19)}$.

Los trabajadores de la salud no están exentos de accidentes ocupacionales aun cuando se ciñan a la aplicación de las normas universales y específicas de bioseguridad. Por este motivo, en los lugares de trabajo deben establecerse planes para hacer frente a las situaciones en las que el trabajador de la salud se lesiona o entra en contacto con sangre en el ejercicio de sus funciones ${ }^{(20)}$. Los agentes biológicos pueden estar presentes en todos los ambientes laborales y algunos son responsables de infecciones, efectos alérgicos, tóxicos y cancerígenos; por lo tanto, deben ser evaluados y controlados para salvaguardar la seguridad y salud del trabajador ${ }^{(21)}$.

\section{REFERENCIAS BIBLIOGRÁFICAS}

1. Vidal J, Basso J, Bagnulo H, Marcolini P. Normas de Bioseguridad del Ministerio de Salud Pública. 1a ed. Uruguay: MSP; 1997; Pp. 6-10.

2. Espinosa Aquino B, Pérez Osorio G , Arriola Morales J, Mendoza Hernández JC, Martínez Tapia I. Bioseguridad, riesgos laborales y protección personal. Rev latinoam ambient cienc. 2010; 1(1): 38-49.

3. Franco A. La seguridad clínica de los pacientes: entendiendo el problema. Colomb Med. 2005; 36(2): 130-133.

4. Manual de bioseguridad de la Universidad Industrial de Santander. Proceso de talento Humano Subproceso Seguridad y Salud Ocupacional, Colombia año 2012; Pág.03.

5. Bautista Rodríguez LM, Delgado Madrid CC, Hernández Zárate ZF, Sanguino Jaramillo FE, Cuevas Santamaría ML, Arias Contreras YT. Nivel de conocimiento y aplicación de las medidas de bioseguridad del personal de enfermería. Rev Cien y Cuid. 2013; 10(2): 127-135.

6. Guía de Bioseguridad para Laboratorios Clínicos. Documentos Técnicos para el laboratorio clínico; Instituto de Salud Pública de Chile. Ministerio de Salud, Gobierno de Chile, 2013, pág. 09.

7. Fink S. Bioseguridad: una responsabilidad del investigador. Rev Medicina Buenos Aires. 2010; 70(3): 299-302.

8. Zayas Bazán S, López Cruz E, Marrero Fente A, Agüero Díaz A. Sida, evaluación de la información sobre las normas de bioseguridad en Estomatología. AMC. 2003;7(6):698-707

9. Del Valle Sol C. Normas de Bioseguridad en el consultorio odontológico. Acta Odontol Venez. 2002; 40(2): 213-216.

10. Organización Mundial de la Salud. Manual de bioseguridad en el laboratorio. 3a ed. Ginebra: OMS; 2005; p. 7

11. Huamán Huaman D, Romero Trujillo L. Nivel de conocimiento y práctica de medidas de bioseguridad en las enfermeras de los servicios de medicina del Hospital Belén de Trujillo 2014. [Tesis para optar el grado de Licenciada en enfermería]. 2014. Universidad Privada Antenor Orrego. Trujillo, Perú. Disponible en: http://repositorio.upao.edu.pe/bitstream/ upaorep/561/1/huaman_doris_medidas_bioseguridad_ enfermeras.pdf

12. Malogon Londoño G, Galán Morera R., Pontón Laverde G. Administración Hospitalaria. 3a Ed. Bogota: Editorial Medica 
Internacional; 2008; pp 171.

13. Vasconez Z., Molina G. Manual de normas de bioseguridad para la red de servicios de salud en el Ecuador. Ministerio de Salud Pública. Ecuador. 2011; Pág.10.

14. Papone V. Normas de Bioseguridad en la práctica odontológica. Ministerio de Salud Pública. Facultad de Odontología. Universidad de la República oriental del Uruguay. 2000. Disponible en: http://files.sld.cu/protesis/files/2011/09/ normas-de-bioseguridad-en-la-practica-odontologica.pdf

15. Ruiz Hernández AR, Fernández García JR. Principios de bioseguridad en los servicios estomatológicos. Medicent Electrón. 2013; 17(2): 49-55.

16. Reeder S, Martín L, Koriak D. Enfermería Materno-Infantil. 17a ed. México: Ediciones McGraw-Hill. 2009; pp:1-134.

17. Organización Mundial de la Salud. Guía de la OMS sobre Higiene de Manos en la Atención de la Salud. OMS: 2009; 07: 13-14. Disponible en: http://www.med.unlp.edu.ar/ archivos/noticias/guia_lavado_de_manos.pdf

18. Ministerio de Salud. Manual de Bioseguridad NT No. 015 MINSA / DGSP - V.01. Sistema de Gestión de la Calidad del Programa Nacional de Hemoterapia y Bancos de Sangre. Lima, Perú 2004. Pág.11

19. Guía de bioseguridad para los profesionales sanitarios, Ministerio de Sanidad, servicios sociales e igualdad, Madrid, Gobierno de España 2015; pág. 29.

20. Manual de Conductas básicas en bioseguridad: manejo integral. Protocolo básico para el equipo de salud. Ministerio de salud. Dirección general de promoción y prevención programa nacional de prevención y control de las ETS/VIH/ SIDA. Santafé de Bogotá. 1997. Pág. 37
21. Mirón A. Directrices para evaluar el riesgo biológico, Centro Nacional de nuevas tecnologías. Instituto Nacional de Seguridad e Higiene del Trabajo, España. 2006. Pp. 02-03.

Fuentes de financiamiento:

Este artículo ha sido financiado por el autor.

Conflicto de interés:

El autor declara no tener ningún tipo de interés.

\section{Correspondencia:}

Frank Dennys Tamariz Chavarria

Dirección: Jirón 27 de julio $N^{\circ} 110$. Callao, Perú. Teléfono: 987557738

Correo electrónico: frank_tamariz@hotmail.com

\section{Recibido: 22 de mayo de 2018 \\ Evaluado: 09 de julio de 2018 \\ Aprobado: 30 de julio de 2018}

( ) La revista. Publicado por Universidad de San Martín de Porres, Perú. (cc) ву Licencia de Creative Commons Artículo en acceso abierto bajo términos de Licencia Creative Commons Atribución 4.0 Internacional. (http://creativecommons.org/licenses/by/4.0/)

ORCID IDS

Frank Dennys Tamariz Chavarria

https://orcid.org/0000-0002-1090-1094 